\title{
INTERVALS IN GENERALIZED EFFECT ALGEBRAS AND THEIR SUB-GENERALIZED EFFECT ALGEBRAS
}

\author{
Zdenka RieČAnOvá*, Michal ZaJAC \\ Department of Mathematics, Faculty of Electrical Engineering and Information Technology STU, Ilkovičova 3, \\ SK-81219 Bratislava \\ * corresponding author: zdenka.riecanova@stuba.sk
}

Abstract. We consider subsets $G$ of a generalized effect algebra $E$ with $0 \in G$ and such that every interval $[0, q]_{G}=[0, q]_{E} \cap G$ of $G(q \in G, q \neq 0)$ is a sub-effect algebra of the effect algebra $[0, q]_{E}$. We give a condition on $E$ and $G$ under which every such $G$ is a sub-generalized effect algebra of $E$.

KEYWORDS: generalized effect algebra, effect algebra, Hilbert space, densely defined linear operators, embedding, positive operators valued state.

\section{INTRODUCTION AND SOME BASIC DEFINITIONS AND FACTS}

The Hilbert space effect algebra $\mathcal{E}(\mathcal{H})$ on a Hilbert space $\mathcal{H}$ is the set of positive operators dominated by the identity operator $I$. In the quantum mechanical framework the elements of an effect algebra represent quantum effects and these are important for quantum statistics and for quantum mechanical theory (see [2, 3]).

One may think of quantum effects as elementary yesno measurements that may be unsharp or imprecise.

Effect algebras were introduced by D. Foulis and M.K. Bennet in 1994 [1]. The prototype for the abstract definition of an effect algebra was the set $\mathcal{E}(\mathcal{H})$ (Hilbert space effects) of all selfadjoint operators between null and identity operators in a complex Hilbert space $\mathcal{H}$. If a quantum mechanical system is represented in the usual way by a complex Hilbert space $\mathcal{H}$ then self-adjoint operators from $\mathcal{E}(\mathcal{H})$ represent yes-no measurements that may be unsharp. Recently several examples and properties of operator (generalized) effect algebras were studied in papers Polakovič, Riečanová [9], Polakovič [10], Paseka, Riečanová [8], Riečanová, Zajac, Pulmannová [12], Pulmannová, Riečanová, Zajac [1], Riečanová, Zajac [13] and Riečanová [14]

The abstract definition of an effect algebra follows the properties of the usual sum of operators in the interval $[0, I]$ (i.e. between null and identity operators in $\mathcal{H})$ and it is the following.

Definition 1.1 (Foulis, Bennet [1]). A partial algebra $(E ; \oplus, 0,1)$ is called an effect algebra if 0,1 are two distinguished elements and $\oplus$ is a partially defined binary operation on $E$ which satisfy the following conditions for any $x, y, z \in E$ :

(E1) $x \oplus y=y \oplus x$ if $x \oplus y$ is defined,

(E2) $(x \oplus y) \oplus z=x \oplus(y \oplus z)$ if one side is defined,

(E3) for every $x \in E$ there exists a unique $y \in E$ such that $x \oplus y=1$ (we put $x^{\prime}=y$ ),
(E4) if $1 \oplus x$ is defined then $x=0$.

Immediately in 1994 the study of generalizations of effect algebras (without the top element 1) was started by several authors (Foulis and Bennet [1], Kalmbach and Riečanová 4, Hedlíková and Pulmannová [5], Kôpka and Chovanec [6]). It was found out that all these generalizations coincide and their common definition is the following:

Definition 1.2. A generalized effect algebra $(E ; \oplus, 0)$ is a set $E$ with element $0 \in E$ and partial binary operation $\oplus$ satisfying for any $x, y, z \in E$ conditions

(GE1) $x \oplus y=y \oplus x$ if one side is defined,

(GE2) $(x \oplus y) \oplus z=x \oplus(y \oplus z)$ if one side is defined,

(GE3) if $x \oplus y=x \oplus z$ then $y=z$,

(GE4) if $x \oplus y=0$ then $x=y=0$,

(GE5) $x \oplus 0=x$ for all $x \in E$.

In every (generalized) effect algebra $E$ a partial order $\leq$ and a binary operation $\ominus$ can be introduced as follows: for any $a, b \in E, a \leq b$ and $b \ominus a=c$ iff $a \oplus c$ is defined and $a \oplus c=b$.

Throughout the paper we assume that $\mathcal{H}$ is an infinite-dimensional complex Hilbert space. For notions and results on Hilbert space operators we refer the reader to [7]. We will assume that the domains $D(A)$ of all considered linear operators $A$ are dense linear subspaces of $\mathcal{H}$ (in the metric topology induced by the inner product). We say that operators $A$ are densely defined in $\mathcal{H}$. The set of all densely defined linear operators on $\mathcal{H}$ will be denoted by $\mathcal{L}(\mathcal{H})$.

Recall that $A: D(A) \rightarrow \mathcal{H}$ is a bounded operator if there exists a real constant $C>0$ such that $\|A x\| \leq$ $C\|x\|$ for all $x \in D(A)$. If $A$ is not bounded then it is called unbounded.

Recall that if $(E ; \oplus, 0,1)$ is an effect algebra $((E ; \oplus, 0)$ is a generalized effect algebra) then a subset $G \neq \emptyset$ such that $1 \in G(0 \in G$ respectively $)$ is a sub-effect algebra (sub-generalized effect algebra) of $E$ iff 
(S) for any $a, b, c \in E$ with $a \oplus b=c$ in $E$ the fact that two out of elements $a, b, c$ are in $G$ implies that all $a, b, c \in G$.

Moreover, as we can easily check, every subgeneralized effect algebra is a generalized effect algebra in its own right.

\section{Sub-GENERALIZED EFFECT ALGEBRAS OF GENERALIZED EFFECT ALGEBRAS}

A significant property of a generalized effect algebra $(E ; \oplus, 0)$ is the fact that for any $q \in E, q \neq 0$, the interval $[0, q]_{E}=\{c \in E \mid$ there exists $d \in E$ such that $c \oplus d=q\}$ is an effect algebra with top element $q$ and the partial binary operation $\oplus_{q}$ defined for $a, b \in[0, q]_{E}$ iff $a \oplus b \leq q$. Then we set $a \oplus_{q} b=a \oplus b$ (we write $\oplus_{q}=\oplus_{[0, q]_{E}}$ ). Thus if a set $G \subseteq E$ with $0 \in G$ is a sub-generalized effect algebra of $E$, then the same is true for all $[0, q]_{E} \cap G$ and $[0, q]_{E}, q \in E$. More precisely,

Theorem 2.1. Let $E$ be a generalized effect algebra and $0 \in G \subseteq E$. Then the following assertions are equivalent:

(1.) $G$ is a sub-generalized effect algebra of $E$.

(2.) For all nonzero $q \in E$ the set $G \cap[0, q]_{E}$ is a sub-generalized effect algebra of $[0, q]_{E}$ considered as a generalized effect algebra.

Proof. (1.) $\Rightarrow(2$.) This implication is obvious.

$(2.) \Rightarrow(1$.$) \quad Let a, b, c \in E, a \oplus b=c$. Substituting $c=q$ into (2) we obtain that $G \cap[0, c]_{E}$ is a subgeneralized effect algebra of $[0, c]_{E}$. Hence $a, b, c \in$ $[0, c]_{E}$ satisfy the property (S), i.e., $G$ is a subgeneralized effect algebra of $E$.

The following example shows that the condition (2.) in Theorem 2.1 cannot be replaced by a stronger one:

(2'.) For all nonzero $q \in E$ the set $G \cap[0, q]_{E}$ is a sub-effect algebra of the effect algebra $[0, q]_{E}$.

Example 2.2. Let $E=\mathbb{R}_{+}$and $G=\mathbb{Q}_{+}$be the sets of all non-negative real and rational numbers, respectively and let + denote the usual sum of real numbers. Then (2) obviously holds but for nonrational $q>0$, e.g. for $q=\sqrt{2}$, we have $G \cap[0, q]_{E}$ is not a sub-effect algebra of $[0, q]_{E}$.

It is easy to see that if $G$ is a sub-generalized effect algebra of a generalized effect algebra $E$, then for all $q \in G, q \neq 0$, the intersection $[0, q]_{G}=[0, q]_{E} \cap G$ is a sub-generalized effect algebra of $[0, q]_{E}$ considered as a generalized effect algebra. Our goal, roughly speaking, is to investigate under what conditions the converse holds.

Theorem 2.3. Let $G$ be a subset of a generalized effect algebra $(E ; \oplus, 0)$ such that $0 \in E$ and for every $c \in E$ there exists $g \in G$ with $c \leq g$. Then the following conditions are equivalent:
(1.) $G$ is a sub-generalized effect algebra of $(E ; \oplus, 0)$.

(2.) For any $q \in G, q \neq 0$ the interval $[0, q]_{G}=$ $[0, q]_{E} \cap G$ in $G$ is a sub-effect algebra of the effect algebra $[0, q]_{E}$.

Proof. (1.) $\Rightarrow$ (2.) This is obvious since (1.) implies that $G$ satisfies the condition $(\mathrm{S})$, hence $[0, q]_{E}$ is an effect algebra for every nonzero $q \in G$. Thus the intersection of two generalized effect algebras $[0, q]_{E} \cap G=[0, q]_{G}$ is an effect algebra, as $q \in G$.

(2.) $\Rightarrow$ (1.) Let $a, b \in G$ with $a \oplus b=c \in E$. There exists $g \in G$ with $c \leq g$ and hence $a \oplus b \in$ $[0, g]_{E} \cap G=[0, g]_{G}$ which, by (2.), gives $a \oplus b \in G$. If $a, c \in G, b \in E$ and $a \oplus b=c$, then by (2.) we have $b \in G$. This proves that $G$ is a sub-generalized effect algebra of $E$.

Example 2.4. Assume that $\mathcal{H}$ is an infinitedimensional complex Hilbert space. Further, let $\mathcal{B}^{+}(\mathcal{H})$ be the set of all bonded positive linear operators with domain $\mathcal{H}$. In 12 it was proved that for any dense linear subspace $D \subseteq \mathcal{H}$ the set $\mathcal{G}_{D}(\mathcal{H})=$ $\mathcal{B}^{+}(\mathcal{H}) \cup\{A: D \rightarrow \mathcal{H} \mid A \geq 0$, unbounded linear operator with $D(A)=D\}$ is a generalized effect algebra with the operation $\oplus_{D}$ which for any $A, B \in \mathcal{G}_{D}(\mathcal{H})$ coincides with the usual sum of linear operators, i.e. $A \oplus_{D} B=A+B$.

It is easy to show that $\mathcal{B}^{+}(\mathcal{H})$ is a sub-generalized effect algebra of $\mathcal{G}_{D}(\mathcal{H})$. For every $Q \in \mathcal{B}^{+}(\mathcal{H}), Q \neq 0$, the intervals under $Q$ in $\mathcal{B}^{+}(\mathcal{H})$ and $\mathcal{G}_{D}(\mathcal{H})$ coincide, i.e. $[0, Q]_{\mathcal{B}^{+}(\mathcal{H})}=[0, Q]_{\mathcal{G}_{D}(\mathcal{H})} \cap \mathcal{B}^{+}(\mathcal{H})=[0, Q]_{\mathcal{G}_{D}(\mathcal{H})}$ and they also coincide as effect algebras. This shows that conditions (1.) and (2.) from Theorem 2.3 hold.

Open Problem 2.5. Example 2.4 shows that, in Theorem 2.3, the condition "to every $q \in E$ there exists $c \in G$ with $q \leq c$ " is only sufficient but not necessary for the equivalence of conditions (1.) and (2.). Thus the open problem remains to find a necessary and sufficient condition for the equivalence of (1.) and (2.) in Theorem 2.3

In fact, for every dense subspace $D$ of $\mathcal{H}$, the generalized effect algebra

$$
\mathcal{G}_{D}(\mathcal{H})=\mathcal{B}^{+}(\mathcal{H}) \cup \mathcal{U}_{D}^{+}(\mathcal{H}),
$$

where $\mathcal{U}_{D}^{+}(\mathcal{H})$ is the set of all unbounded positive linear operators with domain $D$ and the null operator 0 . Clearly, $\mathcal{B}^{+}(\mathcal{H}) \cap \mathcal{U}_{D}^{+}(\mathcal{H})=\{0\}$. On the other hand, while $\mathcal{B}^{+}(\mathcal{H})$ is a sub-generalized effect algebra of $\mathcal{G}_{D}(\mathcal{H})$, the same is not true for $\mathcal{U}_{D}^{+}(\mathcal{H})$. This shows that the union of $\{0\}$ with the difference of two sub-generalized effect algebras of the generalized effect algebra $E$ need not be again a sub-generalized effect algebra of $E$.

Example 2.6. Let $\mathcal{U}_{D}^{+}(\mathcal{H})=\left(\mathcal{G}_{D}(\mathcal{H}) \backslash \mathcal{B}^{+}(\mathcal{H})\right) \cup\{0\}$ be the set of all positive unbounded operators in $\mathcal{H}$ with domain $D$ and the null operator 0 . 
Then $\mathcal{U}_{D}^{+}(\mathcal{H})$ is not a sub-generalized effect algebra of $\mathcal{G}_{D}(\mathcal{H})$ because for $A \in \mathcal{B}^{+}(\mathcal{H})$ and $U, V \in \mathcal{U}_{D}^{+}(\mathcal{H})$ such that $V=U+A$ we have $A \notin \mathcal{U}_{D}^{+}(\mathcal{H})$. It follows that there are $Q \in \mathcal{U}_{D}^{+}(\mathcal{H}), Q \neq 0$, such that $[0, Q]_{\mathcal{U}_{D}^{+}(\mathcal{H})}=[0, Q]_{\mathcal{G}_{D}(\mathcal{H})} \cap \mathcal{U}_{D}^{+}(\mathcal{H})$ is not a sub-effect algebra in $[0, Q]_{\mathcal{G}_{D}(\mathcal{H})}$.

\section{ACKNOWLEDGEMENTS}

This work was supported by grants VEGA 1/0297/11 and VEGA 1/0426/12 of the Ministry of Education of the Slovak Republic and by grant APVV-0178-11 of the Slovak Research and Development Agency.

\section{REFERENCES}

[1] Foulis, D. J., Bennet, M. K.: Effect algebras and unsharp quantum logics, Found. Phys. 24, (1994) 1331-1352.

[2] Foulis, D. J.: Observables, states and symmetries in the context of CB-effect algebras, Reports on Mathematical Physics, 60 (2007), 329-346.

[3] Foulis, D. J.: Effects, observables and symmetries in Physics, Found. Phys. 37 (2007), 1421-1446.

[4] Kalmbach, G., Riečanová, Z.: An axiomatization for abelian relative inverses, Demonstratio Math. 27 (1994), 769-780.

[5] Hedlíková, J., Pulmannová, S.: Generalized difference posets and orthoalgebras, Acta Math. Univ. Comenianae 45 (1996), 247-279.

[6] Kôpka, F., Chovanec, F.: D-posets, Math. Slovaca 44 (1994), 21-34.
[7] Blank, J., Exner, P., Havlíček, M.: Hilbert Space Operators in Quantum Physics (second edition), Springer 2008.

[8] J. Paseka, Z. Riečanová: Considerable sets of linear operators in Hilbert spaces as operator generalized effect algebras, Found. Phys. 41 (2011), 1634-1647.

[9] Polakovič, M., Riečanová, Z.: Generalized effect algebras of positive operators densely defined in Hilbert spaces, Internat. J. Theor. Phys. 50 (2011), 1167-1174.

[10] Polakovič, M.: Generalized effect algebras of bounded positive operators defined on Hilbert spaces, Reports on Mathematical Physics, 68 (2011), 241-250.

[11] Pulmannová, S., Riečanová, Z., Zajac, M.: Topological properties of operator generalized effect algebras, Reports of Mathematical Physics, 69 (2012), No. 2, 311-320.

[12] Riečanová, Z., Zajac, M., Pulmannová, S.: Effect algebras of positive linear operators densely defined on Hilbert spaces, Reports of Mathematical Physics 68 (2011), No. 3, 261-270.

[13] Riečanová, Z., Zajac, M.: Extension of Effect Algebra Operations, Acta Polytechnica 51 (2011) No. 4, 73-77.

[14] Riečanová, Z.: Effect algebra of positive self-adjoint operators densely defined on Hilbert spaces, Acta Polytechnica 51 (2011) No. 4, 78-82.

[15] Riečanová, Z.: Subalgebras, intervals and central elements of generalized effect algebras. International Journal of Theoretic Physics 38, (1999) 3209-3220. 